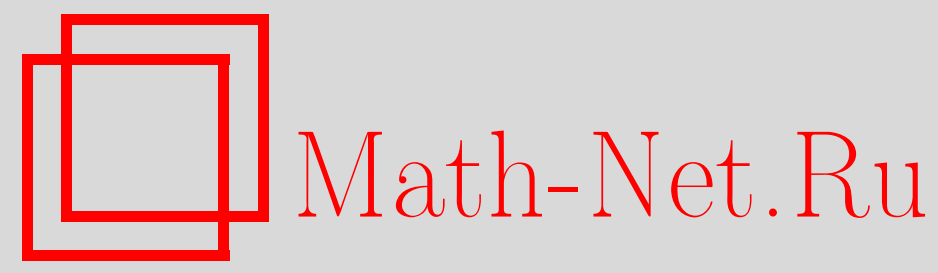

B. Roos, Binomial approximation to the Poisson binomial distribution: The Krawtchouk expansion, Теория вероятн. и ее примен., 2000, том 45, выпуск 2, 328-344

DOI: https://doi.org/10.4213/tvp466

Использование Общероссийского математического портала Math-Net.Ru подразумевает, что вы прочитали и согласны с пользовательским соглашением http://www . mathnet.ru/rus/agreement

Параметры загрузки:

IP: 54.237 .206 .68

26 апреля 2023 г., 15:29:11

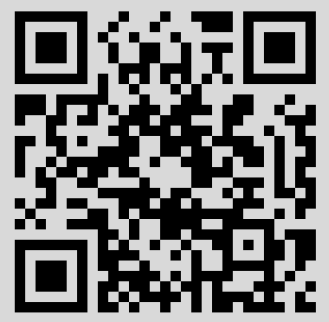


(C) $2000 \mathrm{r}$.

ROOS B.*

\title{
BINOMIAL APPROXIMATION TO THE POISSON BINOMIAL DISTRIBUTION: THE KRAWTCHOUK EXPANSION
}

\begin{abstract}
Биномиальное распределение Пуассона аппроксимируется биномиальным распределением, а также конечными мерами со знаком, получающимися из соответствующего разложения Кравчука. Приведены оценки и асимптотические соотношения для расстояния по вариации и точечной метрики.
\end{abstract}

Ключевые слова и фразы: биномиальная аппрокспмация, биномиальное распределение Пуассона, разложение Кравчука, меры со знаком (заряд), расстояние по вариации, точечная метрика.

\section{Introduction}

1.1. The aim of this paper. We consider the sum $S_{n}$ of $n \in \mathbf{N}=$ $\{1,2, \ldots\}$ independent Bernoulli random variables $X_{1}, \ldots, X_{n}$ with success probabilities

$$
\mathbf{P}\left\{X_{j}=1\right\}=1-\mathbf{P}\left\{X_{j}=0\right\}=p_{j} \in[0,1], \quad j \in\{1, \ldots, n\} .
$$

Since the distribution $P^{S_{n}}$ of $S_{n}$ has a complicated structure, it is often approximated by other distributions. To get higher accuracy, several authors deal with the corresponding asymptotic expansions. We can find publications on normal approximations and the Edgeworth expansion (see [22, Ch. 7] for the binomial case, $[12, \S 4],[5],[13],[23])$ and Poisson approximations and expansions related to the Charlier polynomials (see [14] for the binomial case, $[10],[7, \S 5],[18],[2]-[4],[1],[16],[17])$.

In this paper, we consider the approximation of $P^{S_{n}}$ by the binomial distribution $\mathscr{B}(n, p)$ with parameter $n$ and arbitrary success probability $p$ and also by finite signed measures, resulting from the corresponding Krawtchouk expansion of $P^{S_{n}}$. Here we have to deal with the Krawtchouk polynomials, being orthogonal with respect to the binomial counting density. We prove some bounds and asymptotic relations for the total variation distance $d_{\tau}$ and

${ }^{*}$ Institut für Mathematische Stochastik, Universität Hamburg, Bundesstraße 55, D-20146 Hamburg, Germany; e-mail: roos@math.uni-hamburg.de 
the point metric $d_{\pi}$ between finite signed measures $Q_{1}$ and $Q_{2}$, which are concentrated on $\mathbf{Z}_{+}=\{0,1, \ldots\}$ and satisfy $Q_{1}\left(\mathbf{Z}_{+}\right)=Q_{2}\left(\mathbf{Z}_{+}\right)$:

$$
\begin{aligned}
& d_{\tau}\left(Q_{1}, Q_{2}\right)=\sup _{A \subseteq \mathbf{Z}_{+}}\left|Q_{1}(A)-Q_{2}(A)\right|=\frac{1}{2} \sum_{m=0}^{\infty}\left|Q_{1}(\{m\})-Q_{2}(\{m\})\right|, \\
& d_{\pi}\left(Q_{1}, Q_{2}\right)=\sup _{m \in \mathbf{Z}_{+}}\left|Q_{1}(\{m\})-Q_{2}(\{m\})\right| .
\end{aligned}
$$

The presented method is similar to that used by Shorgin [18] in the Poisson approximation. For refinements of Shorgin's method, see [3], [4], [16], [17].

1.2. Some general notation. In what follows, let

$$
\begin{gathered}
\lambda=\sum_{j=1}^{n} p_{j} \in(0, n), \quad \bar{p}=\frac{\lambda}{n}, \quad \bar{q}=1-\bar{p}, \quad p \in[0,1], \quad q=1-p, \\
\gamma_{k}(p)=\sum_{j=1}^{n}\left(p-p_{j}\right)^{k}, \quad \gamma_{k}=\gamma_{k}(\bar{p}) \quad \text { for } k \in \mathbf{N}, \\
\theta=\frac{\gamma_{2}}{n \bar{p} \bar{q}}=1-\frac{\mathbf{D} S_{n}}{n \bar{p} \bar{q}} .
\end{gathered}
$$

$\mathrm{R}$ e $\mathrm{m}$ a r k. We have $\theta \leqslant \delta \min \left\{1, \delta[4 \bar{p} \bar{q}]^{-1}\right\}$, where $\delta=p_{\max }-p_{\min }$, $p_{\max }=\max _{1 \leqslant j \leqslant n} p_{j}$, and $p_{\min }=\min _{1 \leqslant j \leqslant n} p_{j}$. The proof is given in Section 3.

Further, let

$$
\begin{aligned}
b(m, n, p) & =\Delta^{0} b(m, n, p)=\mathscr{B}(n, p)(\{m\}) \\
& = \begin{cases}\left(\begin{array}{c}
n \\
m
\end{array}\right) p^{m} q^{n-m} & \text { for } n, m \in \mathbf{Z}_{+}, m \leqslant n, \\
0 & \text { otherwise, }\end{cases} \\
\Delta^{j} b(m, n, p) & =\Delta^{j-1} b(m-1, n, p)-\Delta^{j-1} b(m, n, p) \quad \text { for } j \in \mathbf{N} .
\end{aligned}
$$

Here $\left(\begin{array}{l}x \\ m\end{array}\right)=\prod_{k=1}^{m}[(x-k+1) / k]$ for $m \in \mathbf{Z}_{+}$and $x \in \mathbf{C}$. We write $\Delta^{j} b(\cdot, n, q)$ for the sequence $\left(\Delta^{j} b(m, n, q)\right)_{m \in \mathbf{Z}_{+}}$and set $\|f\|_{\infty}=\sup _{m \in \mathbf{Z}_{+}}|f(m)|$ and $\|f\|_{1}=\sum_{m=0}^{\infty}|f(m)|$ for $f: \mathbf{Z}_{+} \rightarrow \mathbf{R}$. Always, let $0^{0}=1$. If not defined otherwise, $i$ is the imaginary unit in C. Let $\lfloor x\rfloor$ be the largest integer $\leqslant x \in \mathbf{R}$.

1.3. Known facts. Ehm $[8$, Theorem 1 , Lemma 2] considered the approximation of $P^{S_{n}}$ by $\mathscr{B}(n, \bar{p})$ and proved the following estimates by using the Stein-Chen method:

$$
\begin{aligned}
\frac{\theta}{124} \min \{1, n \bar{p} \bar{q}\} & \leqslant d_{\tau}\left(P^{S_{n}}, \mathscr{B}(n, \bar{p})\right)=: d \leqslant \frac{1-\bar{p}^{n+1}-\bar{q}^{n+1}}{(n+1) \bar{p} \bar{q}} \gamma_{2} \\
& \leqslant \theta \min \{1, n \bar{p} \bar{q}\} .
\end{aligned}
$$


It follows that $\min \left\{\theta, \gamma_{2}\right\}$ and $d$ are of the same order; further, $d$ is small if and only if $\theta$ is small (see [8, Corollary 2]).

Using the Stein-Chen method, Barbour, Holst, Janson [1, Theorem 9.E] and Soon [19, Corollary 1.3] treated the approximation of $P^{S_{n}}$ by another binomial distribution $\mathscr{B}(\tilde{n}, p)$, where the parameters $\tilde{n} \in \mathbf{N}, p \in(0,1)$ are chosen in such a way that both, $P^{S_{n}}$ and $\mathscr{B}(\tilde{n}, p)$, have the same mean and nearly the same variance. We only cite Soon's result: If $\lambda_{r}=\sum_{j=1}^{n} p_{j}^{r}$ for $r \in \mathbb{N}, \tilde{n}=\left\lfloor\lambda^{2} / \lambda_{2}+\frac{1}{2}\right\rfloor$, and $p=\lambda / \widetilde{n}$, then

$$
d_{\tau}\left(P^{S_{n}}, \mathscr{B}(\widetilde{n}, p)\right) \leqslant \frac{1-p^{\tilde{n}+1}-q^{\tilde{n}+1}}{(\tilde{n}+1) p q}\left[2\left(\lambda_{3}-\frac{\lambda_{2}^{2}}{\lambda}\right)+\lambda\left|p-\frac{\lambda_{2}}{\lambda}\right|\right] .
$$

Note that, if $\tilde{n}=\lambda^{2} / \lambda_{2}$, the variances of $\mathscr{B}(\tilde{n}, p)$ and $S_{n}$ coincide.

Other notes, dealing with binomial approximations, came from $[10$, Section 4$],[12, \S 3],[21$, Section 3$],[9]$, and [11, Corollary 3]. It should be mentioned that Takeuchi and Takemura [21] considered the Krawtchouk expansion of the counting density and the distribution function of the sum of not necessarily independent Bernoulli random variables, whereas Jakševičius [9] approximated the distribution of the sum of independent identically distributed lattice random variables by a binomial type law.

\section{Main results}

2.1. The Krawtchouk expansion of $P^{S_{n}}$.

Theorem 1. For $m \in \mathbf{Z}_{+}$and arbitrary $p$,

$$
\mathbf{P}\left\{S_{n}=m\right\}=\sum_{j=0}^{n} a_{j}(p) \Delta^{j} b(m, n-j, p),
$$

where $a_{0}(p)=1$ and

$$
a_{j}(p)=\sum_{1 \leqslant k(1)<\cdots<k(j) \leqslant n} \prod_{r=1}^{j}\left(p_{k(r)}-p\right), \quad j \in\{1, \ldots, n\} .
$$

We call the right-hand side of (3) the Krawtchouk expansion of $P^{S_{n}}$ with parameter $p$, and $a_{0}(p), \ldots, a_{n}(p)$ the corresponding Krawtchouk coefficients. For $s \in\{0, \ldots, n\}$, the finite signed measure $\mathscr{B}_{s}(n, p)$ concentrated on $\mathbf{Z}_{+}$, is defined by

$$
\mathscr{B}_{s}(n, p)(\{m\})=\sum_{j=0}^{s} a_{j}(p) \Delta^{j} b(m, n-j, p), \quad m \in \mathbf{Z}_{+} .
$$

Note that $\mathscr{B}_{0}(n, p)=\mathscr{B}(n, p)$ and that, for $s \geqslant 1, \mathscr{B}_{s}(n, p)$ depends not only on the indicated arguments but also on $p_{1}, \ldots, p_{n}$, though we omit these 
parameters in our notation. We derive our results for the total variation distance from the inequality

$$
d_{\tau}\left(P^{S_{n}}, \mathscr{B}_{s}(n, p)\right) \leqslant \frac{1}{2} \sum_{j=s+1}^{n}\left|a_{j}(p)\right|\left\|\Delta^{j} b(\cdot, n-j, p)\right\|_{1} .
$$

For the point metric, we use a similar inequality. To get the bounds for the distances [see Theorem 2], we have to estimate the Krawtchouk coefficients of $P^{S_{n}}$ and the norms $\left\|\Delta^{j} b(\cdot, n-j, p)\right\|_{t}$ for $t \in\{1, \infty\}$ [see Lemmas 1,2 , and 4].

$\mathrm{R}$ e m a r k s. 1 . We have $\mathscr{B}_{s}(n, p)\left(\mathbf{Z}_{+}\right)=1$, since $\sum_{m=0}^{\infty} \Delta^{j} b(m, n, p)=$ 0 for $j \in \mathbf{N}$ and $n \in \mathbf{Z}_{+}$. Further, $\mathscr{B}_{s}(n, p)(\{m\})=0$ for $m \in\{n+1$, $n+2, \ldots\}$.

2. By induction over $j$, we get

$$
\frac{d^{j}}{d p^{j}} b(m, n, p)=n^{[j]} \Delta^{j} b(m, n-j, p)
$$

for $n, m \in \mathbf{Z}_{+}, j \in\{0, \ldots, n\}$, with $n^{[j]}=n ! /(n-j) !$, and hence,

$$
\mathscr{B}_{s}(n, p)(\{m\})=\sum_{j=0}^{s} \frac{a_{j}(p)}{n^{[j]}} \frac{d^{j}}{d p^{j}} b(m, n, p), \quad m \in \mathbf{Z}_{+} .
$$

3. With our assumptions, relation (3.5) in [21] is similar to (3).

In the following proposition, we give some alternative formulae for the Krawtchouk coefficients. We need the Krawtchouk polynomials $K_{j}(x, n, p) \in$ $\mathbf{R}[x]$ defined by (see $[20,(2.82 .2)]$ )

$$
K_{j}(x, n, p)=\sum_{k=0}^{j}\left(\begin{array}{c}
n-x \\
j-k
\end{array}\right)\left(\begin{array}{l}
x \\
k
\end{array}\right)(-p)^{j-k} q^{k}, \quad n, j \in \mathbf{Z}_{+}, x \in \mathbf{C} .
$$

Proposition 1. Let $\mu_{(k)}=\sum_{m=k}^{n} m^{[k]} \mathbf{P}\left\{S_{n}=m\right\}$, for $k \in\{0, \ldots, n\}$, be the $k$-th factorial moment of $S_{n}$. Further, let $\alpha \in(0, \infty)$ be arbitrary. Then, for $j \in\{1, \ldots, n\}$,

$$
\begin{aligned}
a_{j}(p) & =\sum_{m=0}^{n} \mathbf{P}\left\{S_{n}=m\right\} K_{j}(m, n, p) \\
& =-\frac{1}{j} \sum_{k=0}^{j-1}\left[a_{k}(p) \gamma_{j-k}(p)\right] \\
& =\sum_{k=0}^{j}\left(\begin{array}{c}
n-k \\
j-k
\end{array}\right) \frac{1}{k !}(-p)^{j-k} \mu_{(k)} \\
& =\frac{1}{2 \pi \alpha^{j}} \int_{0}^{2 \pi} e^{-i j x} \prod_{k=1}^{n}\left[1+\left(p_{k}-p\right) \alpha e^{i x}\right] d x .
\end{aligned}
$$


R e m a r k s. 1. It follows from (10) and (11) that $a_{j}(p)$ can be considered as a function of $\left(\gamma_{1}(p), \ldots, \gamma_{j}(p)\right)$ or of $\left(\mu_{(1)}, \ldots, \mu_{(j)}, p\right)$.

2. Using (10), we get

$$
\begin{aligned}
& a_{1}(p)=-\gamma_{1}(p), \quad a_{2}(p)=\frac{1}{2}\left[\gamma_{1}(p)^{2}-\gamma_{2}(p)\right], \\
& a_{3}(p)=-\frac{1}{6} \gamma_{1}(p)^{3}+\frac{1}{2} \gamma_{1}(p) \gamma_{2}(p)-\frac{1}{3} \gamma_{3}(p),
\end{aligned}
$$

leading to $a_{1}(\bar{p})=0, a_{2}(\bar{p})=-\gamma_{2} / 2$, and $a_{3}(\bar{p})=-\gamma_{3} / 3$.

3. For $m \in \mathbf{Z}_{+}$, we have

$$
\mathscr{B}_{1}(n, p)(\{m\})=b(m, n, p)\left[1-\frac{\gamma_{1}(p)(m-n p)}{n p q}\right],
$$

and, if $n \in\{2,3, \ldots\}$,

$$
\begin{aligned}
& \mathscr{B}_{2}(n, p)(\{m\})=b(m, n, p) \\
& \quad \times\left[1-\frac{\gamma_{1}(p)(m-n p)}{n p q}+\frac{\gamma_{1}(p)^{2}-\gamma_{2}(p)}{2 n(n-1)[p q]^{2}}\right. \\
& \left.\quad \times\left[m^{2}-(1+2(n-1) p) m+n(n-1) p^{2}\right]\right] .
\end{aligned}
$$

Note that $\mathscr{B}(n, \bar{p})=\mathscr{B}_{1}(n, \bar{p})$. For $3 \leqslant s \leqslant n, \mathscr{B}_{s}(n, p)(\{m\})$ can be evaluated by using (8), (10), and (39).

The following proposition shows that the first $s$ moments of $P^{S_{n}}$ and $\mathscr{B}_{s}(n, p)$ coincide.

Proposition 2. For $s \in\{0, \ldots, n\}, k \in\{0, \ldots, s\}$, and $\mu_{(k)}$ as in Proposition 1,

$$
\sum_{m=k}^{n} m^{[k]} \mathscr{B}_{s}(n, p)(\{m\})=\mu_{(k)}
$$

2.2. The bounds and asymptotic relations for the distances. In what follows, we use the notation

$$
\eta(p)=2 \gamma_{2}(p)+\gamma_{1}(p)^{2}, \quad \theta(p)=\frac{\eta(p)}{2 n p q} \text { for } p \in(0,1),
$$

leading to $\eta(\bar{p})=2 \gamma_{2}$ and $\theta(\bar{p})=\theta$.

Theorem 2. Let $s \in\{0, \ldots, n\}, p \in(0,1)$,

$$
\begin{gathered}
C_{1}(s)=\frac{\sqrt{e}(s+1)^{1 / 4}}{2}, \quad C_{2}(s)=\frac{(2 \pi)^{1 / 4} \exp (1 /[24(s+1)]) 2^{(s-1) / 2}}{(s+1)^{1 / 4} \sqrt{s !}} \\
C_{3}(s)=\frac{\sqrt{s+1}}{2}\left(1+\sqrt{\frac{\pi}{2(s+1)}}\right) .
\end{gathered}
$$


Then

$$
\begin{gathered}
d_{\tau}\left(P^{S_{n}}, \mathscr{B}_{s}(n, p)\right) \leqslant C_{1}(s) \theta(p)^{(s+1) / 2} \frac{1-s \sqrt{\theta(p)} /(s+1)}{(1-\sqrt{\theta(p)})^{2}} \\
\text { if } \theta(p)<1, \\
d_{\tau}\left(P^{S_{n}}, \mathscr{B}_{s}(n, p)\right) \leqslant C_{2}(s) \eta(p)^{(s+1) / 2}(1+\sqrt{2 \eta(p)}) \exp (2 \eta(p)), \\
d_{\pi}\left(P^{S_{n}}, \mathscr{B}_{s}(n, p)\right) \leqslant C_{3}(s) \frac{\theta(p)^{(s+1) / 2}(1-s \sqrt{\theta(p)} /(s+1))}{\sqrt{n p q}(1-\sqrt{\theta(p)})^{2}} \\
\text { if } \theta(p)<1 .
\end{gathered}
$$

R e m a r k s. 1. For $p=\bar{p}$ and $s=1$, we get estimates close to the upper bounds from (1).

2. Generally, an inequality $d_{\pi}\left(P^{S_{n}}, \mathscr{B}(n, \bar{p})\right) \leqslant c \theta[n \bar{p} \bar{q}]^{-1 / 2}$ with an absolute constant $c \in(0, \infty)$ cannot hold: If $k \in \mathbf{N}, n=2 k, p_{1}=\cdots=$ $p_{k}=1$, and $p_{k+1}=\cdots=p_{2 k}=0$, then $\bar{p}=\frac{1}{2}$,

$$
d_{\pi}\left(P^{S_{n}}, \mathscr{B}(n, \vec{p})\right) \geqslant\left|\mathbf{P}\left\{S_{n}=k\right\}-b\left(k, 2 k, \frac{1}{2}\right)\right|=\left[1-\frac{(2 k) !}{(k !)^{2} 2^{2 k}}\right] \stackrel{(k \rightarrow \infty)}{\longrightarrow} 1
$$

and $\theta[n \bar{p} \bar{q}]^{-1 / 2}=\sqrt{2 / k} \stackrel{(k \rightarrow \infty)}{\longrightarrow} 0$.

Corollary 1. Let $n \in\{2,3, \ldots\}$ and

$$
x_{ \pm}(n, p)=\left\lfloor\frac{1}{2}+(n-1) p \pm \sqrt{\frac{1}{4}+(n-1) p q}\right\rfloor .
$$

Then $d_{\tau}\left(P^{S_{n}}, \mathscr{B}(n, \bar{p})\right)=H+R$, where

$$
\begin{aligned}
H= & \frac{\left|a_{2}(\bar{p})\right|}{2}\left\|\Delta^{2} b(\cdot, n-2, \bar{p})\right\|_{1} \\
= & \frac{\gamma_{2}}{2(n-1) \bar{p} \bar{q}}\left[\left[x_{+}(n, \bar{p})-(n-1) \bar{p}\right] b\left(x_{+}(n, \bar{p}), n-1, \bar{p}\right)\right. \\
& \left.\quad+\left[(n-1) \bar{p}-x_{-}(n, \bar{p})\right] b\left(x_{-}(n, \bar{p}), n-1, \bar{p}\right)\right] \\
|R| \leqslant & d_{\tau}\left(P^{S_{n}}, \mathscr{B}_{2}(n, \bar{p})\right) \\
\leqslant & \left|\gamma_{3}\right| \min \left\{\frac{\sqrt{3}}{2[n \bar{p} \bar{q}]^{3 / 2}}, \frac{4}{3}\right\} \\
& +\min \left\{1.166 \frac{\theta^{2}(1-3 \sqrt{\theta} / 4)}{(1-\sqrt{\theta})^{2}}, 3.695 \gamma_{2}^{2}\left(1+2 \sqrt{\gamma_{2}}\right) \exp \left(4 \gamma_{2}\right)\right\} .
\end{aligned}
$$

$\mathrm{R}$ e $\mathrm{m}$ a $\mathrm{r} \mathrm{k}$. We have $0 \leqslant x_{-}(n, p) \leqslant(n-1) p \leqslant x_{+}(n, p) \leqslant n-1$.

From the theory of orthogonal polynomials it follows that the zeros of the Krawtchouk polynomials $K_{j}(x, n, p)$ for $n \in \mathbf{N}, j \in\{1, \ldots, n\}$, and 
$p \in(0,1)$, are real, simple, and lie in the interval $(0, n)$. In what follows, we use this fact for $j=3$.

Corollary 2. Let $n \in\{2,3, \ldots\}$ and $0<x_{1}<x_{2}<x_{3}<n+1$ be the zeros of $K_{3}(x, n+1, \bar{p}) \in \mathbf{R}[x]$. Then $d_{\pi}\left(P^{S_{n}}, \mathscr{B}(n, \bar{p})\right)=H^{\prime}+R^{\prime}$, where

$$
\begin{aligned}
H^{\prime}= & \left|a_{2}(\bar{p})\right|\left\|\Delta^{2} b(\cdot, n-2, \bar{p})\right\|_{\infty} \\
= & \frac{\gamma_{2}}{n(n-1)[\bar{p} \bar{q}]^{2}} \max \left\{\left|K_{2}\left(\left\lfloor x_{i}\right\rfloor, n, \bar{p}\right)\right| b\left(\left\lfloor x_{i}\right\rfloor, n, \bar{p}\right) \mid i \in\{1,2,3\}\right\} \\
\left|R^{\prime}\right| \leqslant & d_{\pi}\left(P^{S_{n}}, \mathscr{B}_{2}(n, \bar{p})\right) \\
\leqslant & \left|\gamma_{3}\right| \min \left\{\frac{2.398}{[n \bar{p} \bar{q}]^{2}}, 1\right\} \\
& +\min \left\{\frac{1.627 \theta^{2}(1-3 \sqrt{\theta} / 4)}{\sqrt{n \bar{p} \bar{q}}(1-\sqrt{\theta})^{2}}, 3.695 \gamma_{2}^{2}\left(1+2 \sqrt{\gamma_{2}}\right) \exp \left(4 \gamma_{2}\right)\right\} .
\end{aligned}
$$

Now we present some asymptotic relations. Let us consider the following triangular scheme: We let $n$ and $p_{1}, \ldots, p_{n}$ depend on an additional parameter $k \in \mathbf{N}$ and assume that $k \rightarrow \infty$. Sometimes, we write $\theta^{(k)}$ for $\theta$.

Theorem 3. Let us assume that $\gamma_{2} \neq 0$ for sufficiently large $k$. Set

$$
v=\min \left\{1, \frac{\left|\gamma_{3}\right|}{\gamma_{2} \sqrt{n \bar{p} \bar{q}}}+\frac{1}{n \bar{p} \bar{q}}+\theta\right\}
$$

Then

$$
\begin{gathered}
d_{\tau}\left(P^{S_{n}}, \mathscr{B}(n, \bar{p})\right)=\frac{\theta[1+O(v)]}{\sqrt{2 \pi e}}, \\
d_{\pi}\left(P^{S_{n}}, \mathscr{B}(n, \bar{p})\right)=\frac{\theta[1+O(v)]}{2 \sqrt{2 \pi n \bar{p} \bar{q}}} \text { if } \limsup _{k \rightarrow \infty} \theta^{(k)}<1 .
\end{gathered}
$$

$\mathrm{R}$ e $\mathrm{m}$ a r ks. 1 . Since $\left|\gamma_{3}\right| \leqslant \gamma_{2}$, the preceding asymptotics remain valid if we replace $v$ with $\min \left\{1,[n \bar{p} \bar{q}]^{-1 / 2}+\theta\right\}$.

2. The asymptotics of Theorem 3 have counterparts in the Poisson approximation: Prokhorov [14, Theorem 2] gave an asymptotic formula for the total variation distance between a binomial and a Poisson distribution with the same means. As has been observed in [1, p. 2], the statement of Prokhorov's Theorem 2 contains a minor error. In [1] a new version of it is given, which reads in our notation as follows: $d_{\tau}(\mathscr{B}(n, p), \mathscr{P}(n p)) \leqslant$ $(2 \pi e)^{-1 / 2} p\left[1+O\left(\min \left\{1,[n p]^{-1 / 2}+p\right\}\right)\right]$, where $\mathscr{P}(n p)$ denotes the Poisson distribution with mean $n p$. This relation can be sharpened, if one replaces «s» with « $=\gg$ (see [17]). Generalizations of this result for the Poisson approximation to the Poisson binomial distribution can be found in [2], [3], [16], [17]. 


\section{Proofs}

Proof of the remark in Section 1.2. The first part of the asserted inequality follows from

$$
\gamma_{2}=\sum_{j=1}^{n}\left(\frac{p_{\max }+p_{\min }}{2}-p_{j}\right)\left(\bar{p}-p_{j}\right) \leqslant \frac{\delta}{2} \sum_{j=1}^{n}\left|\bar{p}-p_{j}\right| \leqslant \delta n \bar{p} \bar{q}
$$

since, letting $A_{+}=\left\{j \in\{1, \ldots, n\} \mid p_{j} \geqslant \bar{p}\right\}$ and $A_{-}=\{1, \ldots, n\} \backslash A_{+}$,

$$
\frac{1}{2} \sum_{j=1}^{n}\left|\bar{p}-p_{j}\right|=n \bar{p} \bar{q}-\left(\bar{p} \sum_{j \in A_{+}}\left(1-p_{j}\right)+\bar{q} \sum_{j \in A_{-}} p_{j}\right) \leqslant n \bar{p} \bar{q} .
$$

By using $\gamma_{2} \leqslant \sum_{j=1}^{n}\left(\left(p_{\max }+p_{\min }\right) / 2-p_{j}\right)^{2}$, the remaining part of the inequality is easily shown.

$\mathrm{Pr}$ o of of $\mathrm{Th}$ e or e $\mathrm{m} 1$. We show the assertion by using generating functions and the equality

$$
\sum_{m=0}^{n+j} \Delta^{j} b(m, n, p) z^{m}=[1+p(z-1)]^{n}(z-1)^{j}
$$

for $j, n \in \mathbf{Z}_{+}, z \in \mathbf{C}$, which is easily proved by induction over $j$. For $z \in \mathbf{C}$, we have by independence

$$
\begin{aligned}
\sum_{m=0}^{\infty} \mathbf{P}\left\{S_{n}=m\right\} z^{m} & =\prod_{j=1}^{n}\left[[1+p(z-1)]+\left(p_{j}-p\right)(z-1)\right] \\
& \stackrel{(4)}{=} \sum_{j=0}^{n} a_{j}(p)(z-1)^{j}[1+p(z-1)]^{n-j} \\
& \stackrel{(26)}{=} \sum_{m=0}^{n}\left[\sum_{j=0}^{n} a_{j}(p) \Delta^{j} b(m, n-j, p)\right] z^{m}
\end{aligned}
$$

The proof is completed by comparing the power series.

$\mathrm{Pr}$ o of of Proposition 1. We use the generating functions

$$
\sum_{j=0}^{n} a_{j}(p) z^{j}=\prod_{k=1}^{n}\left[1+\left(p_{k}-p\right) z\right], \quad z \in \mathbf{C},
$$

and, for $n, m \in \mathbf{Z}_{+}, n \geqslant m, z \in \mathbf{C}$,

$$
\sum_{j=0}^{n} K_{j}(m, n, p) z^{j}=[1+q z]^{m}(1-p z)^{n-m} .
$$


Equality (27) is easy to prove, and for (28), see [20, (2.82.4)]. For $z \in \mathbf{C}$, $|z|<1$, we have

$$
\begin{aligned}
& \sum_{j=0}^{n} a_{j}(p) z^{j} \stackrel{(27)}{=}(1-p z)^{n} \prod_{k=1}^{n}\left[1+p_{k}\left(\frac{1+q z}{1-p z}-1\right)\right] \\
&=\sum_{m=0}^{n} \mathbf{P}\left\{S_{n}=m\right\}\left[\frac{1+q z}{1-p z}\right]^{m}(1-p z)^{n} \\
& \stackrel{(28)}{=} \sum_{j=0}^{n}\left[\sum_{m=0}^{n} \mathbf{P}\left\{S_{n}=m\right\} K_{j}(m, n, p)\right] z^{j}, \\
& \sum_{j=1}^{n} j a_{j}(p) z^{j} \stackrel{\stackrel{(27)}{=}}{=}-\left[\sum_{k=1}^{n} \frac{\left(p-p_{k}\right) z}{1-\left(p-p_{k}\right) z}\right] \prod_{k=1}^{n}\left[1+\left(p_{k}-p\right) z\right] \\
&=-\left[\sum_{j=1}^{\infty} \gamma_{j}(p) z^{j}\right]\left[\sum_{j=0}^{n} a_{j}(p) z^{j}\right] \\
&=\sum_{j=1}^{n}\left[-\sum_{k=0}^{j-1} a_{k}(p) \gamma_{j-k}(p)\right] z^{j},
\end{aligned}
$$

and, because of the binomial theorem and $\sum_{k=0}^{n}\left(\mu_{(k)} / k !\right) z^{k}=\prod_{j=1}^{n}\left(1+p_{j} z\right)$,

$$
\begin{aligned}
\sum_{j=0}^{n} a_{j}(p) z^{j} & \stackrel{(27)}{=}(1-p z)^{n} \prod_{j=1}^{n}\left(1+\frac{p_{j} z}{1-p z}\right)=\sum_{k=0}^{n} \frac{\mu_{(k)}}{k !} z^{k}(1-p z)^{n-k} \\
& =\sum_{j=0}^{n}\left[\sum_{k=0}^{j}\left(\begin{array}{c}
n-k \\
j-k
\end{array}\right) \frac{(-p)^{j-k} \mu_{(k)}}{k !}\right] z^{j}
\end{aligned}
$$

from which (9), (10), and (11) follow. Equality (12) follows from (27) and Cauchy's theorem.

Proof of Proposition 2. For $j \in\{0, \ldots, n\}$ and $k \in$ $\{j, \ldots, n\}$, we have

$$
\begin{aligned}
& \left.\sum_{m=k}^{n} \Delta^{j} b(m, n-j, p) m^{[k]} \stackrel{(26)}{=} \frac{d^{k}}{d z^{k}}\left[(1+p(z-1))^{n-j}(z-1)^{j}\right]\right|_{z=1} \\
& \quad=\left.\sum_{i=0}^{k}\left(\begin{array}{c}
k \\
i
\end{array}\right)\left[\frac{d^{k-i}}{d z^{k-i}}(1+p(z-1))^{n-j} \frac{d^{i}}{d z^{i}}(z-1)^{j}\right]\right|_{z=1}=\frac{k !(n-j) ! p^{k-j}}{(k-j) !(n-k) !}
\end{aligned}
$$

Similarly, $\sum_{m=k}^{n} \Delta^{j} b(m, n-j, p) m^{[k]}=0$ for $k \in\{0, \ldots, j-1\}$. Hence

$$
\sum_{m=k}^{n} \mathscr{B}_{s}(n, p)(\{m\}) m^{[k]} \stackrel{(5)}{=} \sum_{j=0}^{k} a_{j}(p) \frac{k !(n-j) ! p^{k-j}}{(k-j) !(n-k) !} \stackrel{(11)}{=} \mu_{(k)}
$$

for $s \in\{0, \ldots, n\}$ and $k \in\{0, \ldots, s\}$, giving the assertion. 
To prove Theorem 2, we need the following four lemmas. Remember that $0^{0}=1$.

Lemma 1. Let $I_{0}(x)=\sum_{m=0}^{\infty}(x / 2)^{2 m} /(m !)^{2}$ be the modified Bessel function of the first kind and order $0, \beta(x)=e^{-x^{2} / 4} I_{0}(x), x \in \mathbf{R}$. Then, for $j \in\{1, \ldots, n\}$ and $\gamma_{2}(p) \neq 0$,

$$
\begin{aligned}
\left|a_{j}(p)\right| \leqslant & {\left[\frac{2 \gamma_{2}(p)+\frac{(n-j)}{n} \gamma_{1}(p)^{2}}{2 j}\right]^{j / 2} \frac{n^{(n-j) / 2}}{(n-j)^{(n-j) / 2}} } \\
& \times \beta\left(\sqrt{\frac{2 j(n-j) \gamma_{1}(p)^{2}}{2 n \gamma_{2}(p)+(n-j) \gamma_{1}(p)^{2}}}\right)
\end{aligned}
$$

P r o of. Let $\alpha, s \in(0, \infty)$ be arbitrary and $j \in\{1, \ldots, n\}$. Using (12), the inequality $1+x \leqslant e^{x}$, and the equality $I_{0}(x)=(2 \pi)^{-1} \int_{0}^{2 \pi} \exp (x \cos y) d y$, $x \in \mathbf{R}$, we get

$$
\begin{aligned}
\left|a_{j}(p)\right| & \leqslant \frac{1}{2 \pi \alpha^{j}} \int_{0}^{2 \pi} \prod_{k=1}^{n}\left|1+\left(p_{k}-p\right) \alpha e^{i x}\right| d x \\
& =\frac{1}{2 \pi \alpha^{j} s^{n / 2}} \int_{0}^{2 \pi} \prod_{k=1}^{n}\left[s+2 s\left(p_{k}-p\right) \alpha \cos x+s\left(p_{k}-p\right)^{2} \alpha^{2}\right]^{1 / 2} d x \\
& \leqslant \frac{\beta\left(s \gamma_{1}(p) \alpha\right)}{\alpha^{j} s^{n / 2}} \exp \left(\frac{n(s-1)}{2}+\frac{\alpha^{2}}{4}\left[2 s \gamma_{2}(p)+s^{2} \gamma_{1}(p)^{2}\right]\right) .
\end{aligned}
$$

Inequality (29) follows in the case $j \neq n$ by letting $\alpha=\left(2 j /\left(2 s \gamma_{2}(p)+\right.\right.$ $\left.\left.s^{2} \gamma_{1}(p)^{2}\right)\right)^{1 / 2}, s=(n-j) / n$. If $j=n$, then (29) follows from the inequality between the arithmetic and geometric means: $\left|a_{n}(p)\right| \stackrel{(4)}{=}\left(\prod_{r=1}^{n}\left(p_{\tau}-p\right)^{2}\right)^{1 / 2} \leqslant$ $\left(\gamma_{2}(p) / n\right)^{n / 2}$. The lemma is proved.

$\mathrm{R}$ e $\mathrm{m}$ a $\mathrm{r} \mathrm{k}$. The inequalities (29), $\beta(x) \leqslant 1$ for $x \in \mathbf{R}$, and $(n-j) / n \leqslant 1$ for $j \in\{1, \ldots, n\}$ lead to the estimate

$$
\left|a_{j}(p)\right| \leqslant\left[\frac{\eta(p)}{2 j}\right]^{j / 2} \frac{n^{(n-j) / 2}}{(n-j)^{(n-j) / 2}}, \quad j \in\{1, \ldots, n\},
$$

which also holds in case of $\gamma_{2}(p)=0$. We will use (30) in the proof of Theorem 2.

Lemma 2. Let $c_{j}=(\sqrt{e} / 2)(1+\sqrt{\pi /(2 j)})$ for $j \in \mathbf{N}$, and $f_{j, n}:[0,1] \rightarrow$ $\mathbf{R}$ with

$$
f_{j, n}(t)=\int_{0}^{\pi / 2}\left[1-t \sin ^{2} x\right]^{n / 2} \sin ^{j} x d x, \quad j, n \in \mathbf{Z}_{+}, t \in[0,1] .
$$

Then

$$
\left\|\Delta^{j} b(\cdot, n, p)\right\|_{\infty} \leqslant \frac{2^{j+1}}{\pi} f_{j, n}(4 p q), \quad j, n \in \mathbf{Z}_{+},
$$


and, for $j \in \mathbf{N}, n \in \mathbf{Z}_{+}, t \in(0,1]$,

$$
f_{j, n}(t) \leqslant \frac{c_{j} \pi}{2^{j+1}}\left(\frac{n}{n+j+1}\right)^{n / 2}\left(\frac{4 j}{(n+j+1) t}\right)^{(j+1) / 2} .
$$

P r o of. Using (26) and Cauchy's theorem, we get

$$
\Delta^{j} b(m, n, p)=\frac{1}{2 \pi} \int_{-\pi}^{\pi} e^{-i x m}\left[1+p\left(e^{i x}-1\right)\right]^{n}\left(e^{i x}-1\right)^{j} d x
$$

for $j, m, n \in \mathbf{Z}_{+}$. By (33), (31) is easily shown. Observe that $4 p q \in[0,1]$. To prove (32), Shorgin's [18, proof of his Lemma 6] inequality

$$
\int_{0}^{\pi / 2} \exp \left(-2 s \sin ^{2} x\right) \sin ^{j} x d x \leqslant c_{j} \pi\left(\frac{j}{4 s e}\right)^{(j+1) / 2}
$$

for $j \in \mathbf{N}, s \in(0, \infty)$, and the estimate $x \leqslant e^{x-1}$ for $x \in \mathbf{R}$ are used: For $t \in(0,1], j, n \in \mathbf{N}$, and arbitrary $y \in(0, \infty)$,

$$
\begin{aligned}
f_{j, n}(t) & =\frac{1}{y^{n / 2}} \int_{0}^{\pi / 2}\left(y-y t \sin ^{2} x\right)^{n / 2} \sin ^{j} x d x \\
& \leqslant \frac{e^{(y-1) n / 2}}{y^{n / 2}} \int_{0}^{\pi / 2} \exp \left(-2\left(\frac{y n t}{4}\right) \sin ^{2} x\right) \sin ^{j} x d x \\
& \leqslant c_{j} \pi g(y) e^{-n / 2}\left(\frac{j}{n t e}\right)^{(j+1) / 2},
\end{aligned}
$$

where $g:(0, \infty) \rightarrow \mathbf{R}, g(y)=e^{n y / 2} y^{-(n+j+1) / 2}$. The function $g$ attains its minimum at $y_{0}=(n+j+1) / n$. Substituting $y=y_{0},(32)$ is shown for $n \neq 0$. In the case $n=0, j \in \mathbf{N}$, and $t \in(0,1]$, we have

$$
f_{j, 0}(t)=\int_{0}^{\pi / 2} \sin ^{j} x d x \leqslant \int_{0}^{\pi / 2} \sin x d x=1 \leqslant c_{j} \pi\left(\frac{j}{(j+1) t}\right)^{(j+1) / 2},
$$

since $[j /(j+1)]^{(j+1) / 2} \geqslant \frac{1}{2}$ and $c_{j} \geqslant \sqrt{e} / 2$ for $j \in \mathbf{N}$. This shows the validity of (32) also in this case and completes the proof.

$\mathrm{R}$ e $\mathrm{m}$ a $\mathrm{r} \mathrm{k}$. Combining the estimates (31) and (32), we get:

$$
\left\|\Delta^{j} b(\cdot, n, p)\right\|_{\infty} \leqslant c_{j}\left(\frac{n}{n+j+1}\right)^{n / 2}\left(\frac{j}{(n+j+1) p q}\right)^{(j+1) / 2}
$$

for $j \in \mathbf{N}, n \in \mathbf{Z}_{+}, p \in(0,1)$, and $c_{j}$ as in Lemma 2 .

The following lemma is needed for Lemma 4.

Lemma 3. For $n \in \mathbf{N}$ and $j \in\{1, \ldots, n\}$,

$$
\frac{n^{n}}{j^{j}(n-j)^{n-j}} \leqslant e \sqrt{j}\left(\begin{array}{l}
n \\
j
\end{array}\right) \text {. }
$$


P r o of. It suffices to prove (36) for $n \in\{2,3, \ldots\}$ and $j \in\{1, \ldots$, $n-1$ \}. For these values, let

$$
T(n, j)=\frac{n^{n} j !(n-j) !}{j^{j}(n-j)^{n-j} n !} \sqrt{\frac{n}{j(n-j)}} .
$$

In what follows, it is shown that $T(n, j) \leqslant[n /(n-1)]^{n-1 / 2}$, from which inequality (36) can be derived. Let $f(x)=[(x+1) / x]^{x+1 / 2}, x \in(0, \infty)$. Since $f(x)$ is decreasing and

$$
\frac{T(n, j)}{T(n, j+1)}=\frac{f(j)}{f(n-j-1)}, \quad n \in\{3,4, \ldots\}, j \in\{1, \ldots, n-2\},
$$

we have $T(n, j) \leqslant T(n, j+1)$ if and only if $n-1 \leqslant 2 j$. Hence

$$
T(n, j) \leqslant \max \{T(n, 1), T(n, n-1)\}=\left(\frac{n}{n-1}\right)^{n-1 / 2},
$$

giving the assertion.

Lemma 4. For $j \in \mathbf{N}, n \in \mathbf{Z}_{+}$, and $p \in(0,1)$,

$$
\begin{aligned}
\left\|\Delta^{j} b(\cdot, n, p)\right\|_{1} & \leqslant\left[\left(\begin{array}{c}
n+j \\
j
\end{array}\right)[p q]^{j}\right]^{-1 / 2} \\
& \leqslant \sqrt{e} j^{1 / 4}\left(\frac{n}{n+j}\right)^{n / 2}\left(\frac{j}{(n+j) p q}\right)^{j / 2} .
\end{aligned}
$$

P r o of. We need the following relations:

$$
\begin{gathered}
K_{j}(m, n+j, p) b(m, n+j, p)=\left(\begin{array}{c}
n+j \\
j
\end{array}\right)[p q]^{j} \Delta^{j} b(m, n, p), \\
\sum_{m=0}^{n} b(m, n, p) K_{j}(m, n, p) K_{r}(m, n, p)=\delta_{j, r}\left(\begin{array}{l}
n \\
j
\end{array}\right)[p q]^{j}
\end{gathered}
$$

for $m, n, j, r \in \mathbf{Z}_{+}$and $p \in[0,1]$. Here $\delta_{j, r}$ is the Kronecker symbol. Identity (39) follows from (26) and

$$
\sum_{m=0}^{n+j} K_{j}(m, n+j, p) b(m, n+j, p) z^{m} \stackrel{(8)}{=}\left(\begin{array}{c}
n+j \\
j
\end{array}\right)[p q]^{j}(1+p(z-1))^{n}(z-1)^{j} .
$$

For (40), see $[20,(2.82 .6)]$. The inequalities (37) and (38) follow from (39), (40), (36), and Cauchy's inequality:

$$
\begin{gathered}
\left\|\Delta^{j} b(\cdot, n, p)\right\|_{1}=\left[\left(\begin{array}{c}
n+j \\
j
\end{array}\right)[p q]^{j}\right]^{-1} \sum_{m=0}^{n+j} b(m, n+j, p)\left|K_{j}(m, n+j, p)\right| \\
\leqslant\left[\left(\begin{array}{c}
n+j \\
j
\end{array}\right)[p q]^{j}\right]^{-1 / 2} \leqslant e^{1 / 2} j^{1 / 4}\left(\frac{n}{n+j}\right)^{n / 2}\left(\frac{j}{(n+j) p q}\right)^{j / 2} .
\end{gathered}
$$

The lemma is proved. 
P r o o f of $\mathrm{T} \mathrm{h}$ e o r e m 2. It suffices to prove the assertion in the case $s \leqslant n-1$. Using (3), (30), (38), and the inequality $j^{1 / 4} \leqslant j(s+1)^{-3 / 4}$ for $j \geqslant s+1$, we obtain in the case $\theta(p)<1$,

$$
\begin{aligned}
d_{\tau}\left(P^{S_{n}}, \mathscr{B}_{s}(n, p)\right) & \leqslant \frac{1}{2} \sum_{j=s+1}^{n}\left|a_{j}(p)\right|\left\|\Delta^{j} b(\cdot, n-j, p)\right\|_{1} \\
& \leqslant \frac{\sqrt{e}}{2} \sum_{j=s+1}^{n} \theta(p)^{j / 2} j^{1 / 4} \\
& \leqslant C_{1}(s) \theta(p)^{(s+1) / 2} \frac{1-s \sqrt{\theta(p)} /(s+1)}{(1-\sqrt{\theta(p)})^{2}},
\end{aligned}
$$

giving (15). To prove (16), we use (3), (30), the inequality

$$
\left\|\Delta^{j} b(\cdot, n, p)\right\|_{1} \leqslant 2^{j}, \quad j, n \in \mathbf{Z}_{+}, p \in[0,1],
$$

Stirling's formula (see $[6$, p. 54])

$$
j !=\sqrt{2 \pi} j^{j+1 / 2} \exp \left(\vartheta_{j}-j\right), \quad \vartheta_{j} \in\left[\frac{1}{12 j+1}, \frac{1}{12 j}\right], \quad j \in \mathbf{N},
$$

and the inequalities $1+x \leqslant e^{x}$ for $x \in \mathbf{R}$, and

$$
\sum_{j=m}^{\infty} \frac{x^{j}}{\sqrt{j !}} \leqslant \frac{x^{m}}{\sqrt{m !}} \sum_{j=0}^{\infty} \frac{x^{j}}{\sqrt{j !}}\left(\begin{array}{c}
j \\
\lfloor j / 2\rfloor
\end{array}\right)^{1 / 2} \leqslant \frac{x^{m}}{\sqrt{m !}}(1+x) \exp \left(x^{2}\right)
$$

for $x \in[0, \infty), m \in \mathbf{Z}_{+}$. We get

$$
\begin{aligned}
d_{\tau}\left(P^{S_{n}}, \mathscr{B}_{s}(n, p)\right) & \leqslant \frac{1}{2} \sum_{j=s+1}^{n}\left|a_{j}(p)\right| 2^{j} \leqslant \frac{1}{2} \sum_{j=s+1}^{\infty}\left(\frac{2 \eta(p) e}{j}\right)^{j / 2} \\
& \leqslant \frac{(2 \pi)^{1 / 4} \exp (1 /[24(s+1)])}{2} \sum_{j=s+1}^{\infty} \frac{(2 \eta(p))^{j / 2}}{\sqrt{j !}} j^{1 / 4} \\
& \leqslant C_{2}(s) \eta(p)^{(s+1) / 2}(1+\sqrt{2 \eta(p)}) \exp (2 \eta(p)) .
\end{aligned}
$$

The proof of (17) is similar. Here we use (3), (30), (35), and the inequalities $1+x \leqslant e^{x}$ for $x \in \mathbf{R}$ and $j^{1 / 2} \leqslant j(s+1)^{-1 / 2}$ for $j \geqslant s+1$.

Proof of Corolla r y 1. Only (19) and (20) require a proof. We have

$$
d_{\tau}\left(P^{S_{n}}, \mathscr{B}_{2}(n, \bar{p})\right) \leqslant \frac{1}{2}\left|a_{3}(\bar{p})\right|\left\|\Delta^{3} b(\cdot, n-3, \bar{p})\right\|_{1}+d_{\tau}\left(P^{S_{n}}, \mathscr{B}_{3}(n, \bar{p})\right)
$$

if $n \geqslant 3$, and $R=0$ in case of $n=2$. Inequality (20) can be shown by using Theorem 2, (41), $a_{3}(\bar{p})=-\gamma_{3} / 3$ and, in case of $n \geqslant 3$,

$$
\left\|\Delta^{3} b(\cdot, n-3, \bar{p})\right\|_{1} \stackrel{(37)}{\leqslant}\left[\left(\begin{array}{l}
n \\
3
\end{array}\right)[\bar{p} \bar{q}]^{3}\right]^{-1 / 2} \leqslant\left(\frac{3}{n \bar{p} \bar{q}}\right)^{3 / 2} .
$$

Identity (19) follows from $a_{2}(\bar{p})=-\gamma_{2} / 2$ and the next lemma. 
Lemma 5. Let $n \in\{2,3, \ldots\}, p \in(0,1)$, and $x_{ \pm}(n, p)$ as in Corollary 1. Then

$$
\begin{aligned}
\left\|\Delta^{2} b(\cdot, n-2, p)\right\|_{1}= & \frac{2}{(n-1) p q}\left[\left[x_{+}(n, p)-(n-1) p\right] b\left(x_{+}(n, p), n-1, p\right)\right. \\
& \left.+\left[(n-1) p-x_{-}(n, p)\right] b\left(x_{-}(n, p), n-1, p\right)\right] .
\end{aligned}
$$

$\mathrm{P}_{\mathrm{r}}$ o o f. Let $v_{m}=\Delta^{2} b(m, n-2, p)$ for $m \in \mathbf{Z}_{+}$. Observe that $x_{ \pm}(n, p)$ are the integer parts of the zeros of $K_{2}(x, n, p)=\frac{1}{2}\left[x^{2}-(1+2(n-1) p) x+\right.$ $\left.n(n-1) p^{2}\right] \in \mathbf{R}[x]$, and hence,

$$
\begin{aligned}
\left\|\left(v_{m}\right)_{m \in \mathbf{Z}_{+}}\right\|_{1} & \stackrel{(39)}{=}\left(\begin{array}{l}
n \\
2
\end{array}\right)^{-1}[p q]^{-2} \sum_{m=0}^{n}\left|K_{2}(m, n, p)\right| b(m, n, p) \\
& =\sum_{m=0}^{x_{-}(n, p)} v_{m}-\sum_{m=x_{-}(n, p)+1}^{x_{+}(n, p)} v_{m}+\sum_{m=x_{+}(n, p)+1}^{\infty} v_{m} \\
& =2\left[\Delta^{1} b\left(x_{+}(n, p), n-2, p\right)-\Delta^{1} b\left(x_{-}(n, p), n-2, p\right)\right] .
\end{aligned}
$$

By using (39), the proof is completed.

Corollary 2 is easily proved by using the following lemma.

Lemma 6. Let $n \in\{2,3, \ldots\}, p \in(0,1)$, and $0<x_{1}<x_{2}<x_{3}<n+1$ be the zeros of $K_{3}(x, n+1, p) \in \mathbf{R}[x]$. Then

$$
\begin{aligned}
& \left\|\Delta^{2} b(\cdot, n-2, p)\right\|_{\infty} \\
& \quad=\frac{2}{n(n-1)[p q]^{2}} \max \left\{\left|K_{2}\left(\left\lfloor x_{i}\right\rfloor, n, p\right)\right| b\left(\left\lfloor x_{i}\right\rfloor, n, p\right) \mid i \in\{1,2,3\}\right\} .
\end{aligned}
$$

P r o o f. For $m \in\{0, \ldots, n-1\}$ and $v_{m}$ as in the proof of Lemma 5,

$$
\begin{aligned}
v_{m} \leqslant v_{m+1} & \Longleftrightarrow \Delta^{3}(m+1, n-2, p) \leqslant 0 \stackrel{(39)}{\Longleftrightarrow} K_{3}(m+1, n+1, p) \leqslant 0 \\
& \Longleftrightarrow\left[0 \leqslant m+1 \leqslant x_{1} \text { or } x_{2} \leqslant m+1 \leqslant x_{3}\right], \\
v_{m} \geqslant v_{m+1} & \Longleftrightarrow\left[x_{1} \leqslant m+1 \leqslant x_{2} \text { or } x_{3} \leqslant m+1 \leqslant n\right] .
\end{aligned}
$$

Hence, letting $x_{0}=0$ and $x_{4}=n$,

$$
\sup _{m \in \mathbf{Z}_{+}} v_{m}=\max _{i \in\{1,3\}} v_{\left\lfloor x_{i}\right\rfloor}, \quad \inf _{m \in \mathbf{Z}_{+}} v_{m}=\min _{i \in\{0,2,4\}} v_{\left\lfloor x_{i}\right\rfloor} .
$$

This yields

$$
\sup _{m \in \mathbf{Z}_{+}}\left|v_{m}\right|=\max _{i \in\{0, \ldots, 4\}}\left|v_{\left\lfloor x_{i}\right\rfloor}\right| .
$$

Since $0 \leqslant v_{0} \leqslant v_{\left\lfloor x_{1}\right\rfloor}$ and $0 \leqslant v_{n} \leqslant v_{\left\lfloor x_{3}\right\rfloor}$, the assertion follows from (39).

For the proof of Theorem 3, we need the following two lemmas. 
Lemma 7. Let $p \in(0,1), n \in \mathbf{N}$, and $m \in\{0, \ldots, n\}$. Further, let $x=(m-n p)[n p q]^{-1 / 2}$, satisfying $|x| \leqslant A$, where $A \in(0, \infty)$ is an absolute constant. Then

$$
b(m, n, p)=\frac{e^{-x^{2} / 2}}{\sqrt{2 \pi n p q}}\left[1+\frac{\left(x^{3}-3 x\right)(q-p)}{6 \sqrt{n p q}}+O\left(\frac{1}{n p q}\right)\right],
$$

where the constant intervening in $O\left([n p q]^{-1}\right)$ depends on $A$ only.

$\mathrm{Pr}$ o of. See $[22$, p. 135 , problem 7$]$ or, in case of fixed $p,[15$, p. 151 , Theorem 1].

Lemma 8. For $p \in(0,1)$ and $n \in\{2,3, \ldots\}$,

$$
\begin{aligned}
\left\|\Delta^{2} b(\cdot, n-2, p)\right\|_{1} & =\frac{4}{\sqrt{2 \pi e} n p q}\left[1+O\left(\frac{1}{n p q}\right)\right], \\
\left\|\Delta^{2} b(\cdot, n-2, p)\right\|_{\infty} & =\frac{1}{\sqrt{2 \pi}[n p q]^{3 / 2}}\left[1+O\left(\frac{1}{n p q}\right)\right] .
\end{aligned}
$$

P r o o f. Since $\left\|\Delta^{2} b(\cdot, n-2, p)\right\|_{1} \leqslant 4$, we may assume $n p q \rightarrow \infty$. First, we prove (44) by using Lemma 5 . Let $n \in\{2,3, \ldots\}, p \in(0,1), x_{ \pm}(n, p)$ as in Corollary 1 , and $y_{ \pm}(n, p)=\left(x_{ \pm}(n, p)-(n-1) p\right)[(n-1) p q]^{-1 / 2}$. For simplicity, we omit the indicated arguments for $x_{ \pm}$and $y_{ \pm}$. Using Lemma 7 and the relations

$$
\begin{gathered}
y_{ \pm} \mp 1= \\
O\left(\frac{1}{\sqrt{n p q}}\right), \quad e^{-y_{ \pm}^{2} / 2}=\frac{2 \mp y_{ \pm}}{\sqrt{e}}+O\left(\frac{1}{n p q}\right), \\
\frac{1}{\sqrt{(n-1) p q}}=\frac{1}{\sqrt{n p q}}+O\left(\frac{1}{[n p q]^{3 / 2}}\right)
\end{gathered}
$$

we get

Since

$$
b\left(x_{ \pm}, n-1, p\right)=\frac{2 \mp y_{ \pm}}{\sqrt{2 \pi e n p q}}+\frac{\left(y_{ \pm}^{3}-3 y_{ \pm}\right)(q-p)}{6 \sqrt{2 \pi e} n p q}+O\left(\frac{1}{[n p q]^{3 / 2}}\right) .
$$

$$
x_{ \pm}-(n-1) p=y_{ \pm}\left(\sqrt{n p q}+O\left(\frac{1}{\sqrt{n p q}}\right)\right), \quad\left|y_{+}\right|-\left|y_{-}\right|=O\left(\frac{1}{\sqrt{n p q}}\right)
$$

it follows that

$$
\begin{aligned}
& {\left[x_{+}-(n-1) p\right] b\left(x_{+}, n-1, p\right)+\left[(n-1) p-x_{-}\right] b\left(x_{-}, n-1, p\right)} \\
& \quad=\frac{2}{\sqrt{2 \pi e}}+\frac{\left[\left(y_{+}^{4}-y_{-}^{4}\right)-3\left(y_{+}^{2}-y_{-}^{2}\right)\right](q-p)}{6 \sqrt{2 \pi e n p q}}+O\left(\frac{1}{n p q}\right) \\
& =\frac{2}{\sqrt{2 \pi e}}+O\left(\frac{1}{n p q}\right),
\end{aligned}
$$

giving relation (44). Now we prove (45). For $j \in \mathbf{Z}_{+}, n \in \mathbf{N}, t \in(0,1]$, and $f_{j, n}(t)$ as in Lemma 2 , we have

$$
f_{j, n}(t) \leqslant f_{j+2, n}(t)+\frac{1}{2} \Gamma\left(\frac{j+1}{2}\right)\left(\frac{2}{n t}\right)^{(j+1) / 2}
$$


since, by using $\sin ^{2} x+\cos ^{2} x=1, x \in \mathbf{R}$, we get $f_{j, n}(t)=f_{j+2, n}(t)+I_{j, n}(t)$, where

$$
\begin{aligned}
I_{j, n}(t) & =\int_{0}^{\pi / 2}\left[1-t \sin ^{2} x\right]^{n / 2} \sin ^{j} x \cos ^{2} x d x \\
& =\int_{0}^{t} \frac{[1-y]^{n / 2} y^{(j-1) / 2}}{2 t^{(j+1) / 2}} \sqrt{1-\frac{y}{t}} d y \leqslant \frac{1}{2 t^{(j+1) / 2}} \int_{0}^{t} e^{-n y / 2} y^{(j-1) / 2} d y \\
& \leqslant \frac{1}{2} \Gamma\left(\frac{j+1}{2}\right)\left(\frac{2}{n t}\right)^{(j+1) / 2} .
\end{aligned}
$$

With $j=2, n \geqslant 3$, and $p \in(0,1)$, it easily follows from (31), (46), and (32) that, for an absolute constant $M \in(0, \infty)$,

$$
\left\|\Delta^{2} b(\cdot, n-2, p)\right\|_{\infty} \leqslant \frac{1}{\sqrt{2 \pi}[n p q]^{3 / 2}}+\frac{M}{[n p q]^{5 / 2}} .
$$

To prove (45), we show a similar lower bound. We have

$$
\left\|\Delta^{2} b(\cdot, n-2, p)\right\|_{\infty} \geqslant\left|\Delta^{2} b(\lfloor n p\rfloor, n-2, p)\right| \stackrel{(39)}{=} T_{1} T_{2} T_{3},
$$

where, letting $r=n p-\lfloor n p\rfloor$,

$$
\begin{aligned}
& T_{1}=\frac{2}{n(n-1)[p q]^{2}}=\frac{2}{[n p q]^{2}}+O\left(\frac{1}{[n p q]^{3}}\right), \\
& T_{2}=\left|K_{2}(\lfloor n p\rfloor, n, p)\right|=\frac{1}{2}|n p q+r(p-q-r)|=\frac{n p q}{2}+O(1), \\
& T_{3}=b(\lfloor n p\rfloor, n, p)=\frac{1}{\sqrt{2 \pi n p q}}+O\left(\frac{1}{[n p q]^{3 / 2}}\right) .
\end{aligned}
$$

For the latter bound, Lemma 7 has been used. Hence

$$
T_{1} T_{2} T_{3}=\frac{1}{\sqrt{2 \pi}[n p q]^{3 / 2}}+O\left(\frac{1}{[n p q]^{5 / 2}}\right)
$$

leading to

$$
\left\|\Delta^{2} b(\cdot, n-2, p)\right\|_{\infty} \geqslant \frac{1}{\sqrt{2 \pi}[n p q]^{3 / 2}}-\frac{M^{\prime}}{[n p q]^{5 / 2}}
$$

for an absolute constant $M^{\prime} \in(0, \infty)$ and sufficiently large $n p q$. The lemma is proved.

P r o of of $\mathrm{T}$ h e or e m 3. In the case $\lim \sup _{k \rightarrow \infty} \theta^{(k)}<1$, the relations (24) and (25) are easily shown by using Corollaries 1, 2, Lemma 8 , and the inequalities (15), (17) for $s=1, \bar{p}=p$. Since

$$
d_{\tau}\left(P^{S_{n}}, \mathscr{B}(n, \bar{p})\right) \leqslant 1,
$$

the above condition for (24) can be dropped.

I thank Lutz Mattner and the referee for their useful remarks. 


\section{REFERENCES}

1. Barbour A. D., Holst L., Janson S. Poisson Approximation. Oxford: Clarendon Press, $1992,277 \mathrm{p}$.

2. Deheuvels $P$., Pfeifer $D$. A semigroup approach to Poisson approximation. - Ann. Probab., 1986, v. 14, p. 663-676.

3. Deheuvels $P$., Pfeifer $D$. On a relationship between Uspensky's theorem and Poisson approximations. - Ann. Inst. Statist. Math., 1988, v. 40, p. 671-681.

4. Deheuvels P., Pfeifer D., Puri M.L. A new semigroup technique in Poisson approximation. - Semigroup Forum, 1989, v. 38, p. 189-201.

5. Deheuvels P., Puri M. L., Ralescu S.S. Asymptotic expansions for sums of nonidentically distributed Bernoulli random variables. - J. Multivariate Anal., 1989, v. 28, p. 282-303.

6. Feller $W$. An Introduction to Probability Theory and its Applications, I. New York: Wiley, 1968, $509 \mathrm{p}$.

7. Franken $P$. Approximation der Verteilungen von Summen unabhängiger nichtnegativer ganzzahliger Zufallsgrößen durch Poissonsche Verteilungen. - Math. Nachr., 1964, v. 27 , p. 303-340.

8. Ehm W. Binomial approximation to the Poisson binomial distribution. - Statist. Probab. Lett., 1991, v. 11, p. 7-16.

9. Якиявичус IIT. В. Приближение законом биномиального типа. - Литов. матем. c6., 1992, т. 32, Ne 2, p. 221-228.

10. Le Cam $L$. An approximation theorem for the Poisson binomial distribution. Pacific J. Math., 1960, v. 10, p. 1181-1197.

11. Loh W.-L. Stein's method and multinomial approximation. - Ann. Appl. Probab., 1992 , v. 2, p. 536-554.

12. Makabe $H$. On approximations to some limiting distributions with applications to the theory of sampling inspections by attributes. - Kodai Math. Semin. Rep., 1964, v. 16, p. 1-17.

13. Михайлов В.Г. Об уточнении центральной предельной теоремы для суммы независимых случайных индикаторов. - Теория вероятн. и ее примен., 1993, т. 38, B. 3, c. 540-552.

14. Прохоров ЮО.В. Асимптотическое поведение биномиального распределения. Успехи матем. наук, 1953, т. 8, в. 3, с. 135-142.

15. Rényi A. Probability Theory. Amsterdam: North-Holland Publishing, 1970, 666 p.

16. Roos B. A semigroup approach to Poisson approximation with respect to the point metric. - Statist. Probab. Lett., 1995, v. 24, p. 305-314.

17. Roos $B$. Asymptotics and sharp bounds in the Poisson approximation to the Poisson binomial distribution. - Bernoulli, 1999, v. 5, p. 1021-1034.

18. Шорәия С. Я. Аппроксимация обобценного биномиального распределения. - Теория вероятн. и ее примен., 1977 , т. 22 , в. 4, с. 867-871.

19. Soon S. Y.T. Binomial approximation for dependent indicators. - Statist. Sinica, 1996 , v. 6, p. 703-714.

20. Szegö G. Orthogonal Polynomials. Providence, RI: Amer. Math. Soc., 1975, 432 p.

21. Takeuchi $K$., Takemura A. On sum of 0-1 random variables. I. Univariate case. Ann. Inst. Statist. Math., 1987, v. 39, p. 85-102.

22. Uspensky J. V. Introduction to Mathematical Probability. New York: McGraw-Hill, 1937, $411 \mathrm{p}$.

23. Волкова А. Ю. Уточнение центральной предельной теоремы для сумм независимых случайных индикаторов. - Теория вероятн. и ее примен., 1995, т. 40, в. 4, c. $885-888$. 